\title{
A CA Hybrid of the Slow-to-Start and the Optimal Velocity Models and its Flow-Density Relation*
}

\author{
Hideaki UJINO ${ }^{\dagger}$ and Tetsu YAJIMA ${ }^{\ddagger}$ \\ $\dagger$ National Institute of Technology, Gunma College, Maebashi, Gunma 371-8530, Japan \\ E-mail: ujino@nat.gunma-ct.ac.jp \\ ¥ Department of Information Systems Science, Graduate School of Engineering, \\ Utsunomiya University, Utsunomiya 321-8585, Japan \\ E-mail: yajimat@is.utsunomiya-u.ac.jp
}

Received March 31, 2015, in final form July 27, 2015; Published online July 31, 2015

http://dx.doi.org/10.3842/SIGMA.2015.065

\begin{abstract}
The s2s-OVCA is a cellular automaton (CA) hybrid of the optimal velocity $(\mathrm{OV})$ model and the slow-to-start (s2s) model, which is introduced in the framework of the ultradiscretization method. Inverse ultradiscretization as well as the time continuous limit, which lead the s2s-OVCA to an integral-differential equation, are presented. Several traffic phases such as a free flow as well as slow flows corresponding to multiple metastable states are observed in the flow-density relations of the s2s-OVCA. Based on the properties of the stationary flow of the s2s-OVCA, the formulas for the flow-density relations are derived.
\end{abstract}

Key words: optimal velocity (OV) model; slow-to-start (s2s) effect; cellular automaton (CA); ultradiscretization, flow-density relation

2010 Mathematics Subject Classification: 39A10; 39A06

\section{Introduction}

Self-driven many-particle systems have provided a good microscopic point of view on the vehicle traffic $[3,5]$. The optimal velocity model [1] gives a description of such a system with a set of ordinary differential equations (ODE). It is a car-following model describing an adaptation to the optimal velocity that depends on the distance from the vehicle ahead. Another way of describing such systems is provided by cellular automata (CA). For example, the elementary CA of Rule 184 (ECA184) [16], the Fukui-Ishibashi (FI) model [4] and the slow-to-start (s2s) model [12] are CA describing vehicle traffic as self-driven many-particle systems.

Studies of the self-driven many-particle systems have been wanting a framework that commands a bird's eye view of both ODE and CA models in a unified manner. Ultradiscretization [14], which gives a link between the Korteweg-de Vries (KdV) equation and integrable soliton CA [11], is expected to provide such a framework, for it can be applied to non-integrable systems, too. As a first step toward such a framework, an ultradiscretization of the OV model [10] was presented. A specific choice of the OV function enabled the ultradiscretization of the OV model without any other specialization. We should note that another ultradiscretization of the OV model with essentially the same OV function as above was derived from the modified KdV $(\mathrm{mKdV})$ equation, which is an effective theory around the critical point, with specializing its solutions to traveling wave solutions [7]. The latter ultradiscretization of the OV model depends on the ultradiscretization of the mKdV equation, which is an integrable soliton equation with rich accumulation of the studies of integrable discretization and ultradiscretization. The former

\footnotetext{
${ }^{\star}$ This paper is a contribution to the Special Issue on Exact Solvability and Symmetry Avatars in honour of Luc Vinet. The full collection is available at http://www.emis.de/journals/SIGMA/ESSA2014.html
} 
one, on the other hand, has nothing to do with integrability, which indicates a possibility to expand the scope of the ultradiscretization beyond the integrable models. Thus the two ultradiscretizations make a clear contrast regarding to integrability. An early search for a CA-type OV model dates back to 1999 [5, 6], which was done from a phenomenological point of view to highway traffic.

The former ultradiscretization of the OV model [10] provided a foundation toward a hybridization of the OV model and the s2s model, and it lead to the s2s-OVCA [9] indeed, without the aid of the integrable models. The s2s-OVCA is a CA-type hybrid of the OV model and the s2s model. As we shall see, the equation of the s2s-OVCA generally involves three or more times, or higher order time-differences, in other words. As far as the authors know, difference equations involving higher order time-differences yet want a thorough study from a point of view of the ultradiscretization. Besides an interest from the traffic theoretical point of view, an interest toward a new horizon of the scope of the ultradiscretization motivates us to introduce and study the s2s-OVCA. As we shall show in Section 2, the s2s-OVCA reduces to an ODE that is an extension of the OV model in the inverse-ultradiscrete and the time-continuous limits.

It was observed by numerical experiments that motion of the vehicles described by the s2sOVCA went to stationary flow in the long run, irrespectively of the initial configuration $[9,15]$. It was also observed by numerical experiments that the flow-density relation for the stationary flow of the s2s-OVCA was piecewise linear and flipped- $\lambda$ shaped with several metastable slow branches [9]. Exact expression for the flow-density relation was given by a set of exact solutions giving stationary flows of the s2s-OVCA [15]. The flipped- $\lambda$ shaped diagram captures the characteristic of observed flow-density relations [3,5]. Some other CA type models that gave a flipped- $\lambda$ shaped flow-density relation with a metastable branch was also reported [2]. We shall explain in Section 3 the flow-density relation of the s2s-OVCA based on the properties of the stationary flow which was numerically observed [9].

\section{$2 \quad$ s2s-OVCA and its inverse ultradiscretization}

The s2s-OVCA is given by a set of difference equations below

$$
x_{k}^{n+1}=x_{k}^{n}+\min \left(\min _{n^{\prime}=0}^{n_{0}}\left(x_{k+1}^{n-n^{\prime}}-x_{k}^{n-n^{\prime}}-1\right), v_{0}\right),
$$

where the integers $n_{0} \geq 0, v_{0} \geq 0$ and $x_{k}^{n}, k=1,2, \ldots, K$, are the monitoring period, the top speed and the position of the car $k$ at the $n$-th discrete time. Note that the definition of the symbol $\min _{k=0}^{N}$ is

$$
\min _{k=0}^{N}\left(a_{k}\right):=\min \left(a_{0}, a_{1}, a_{2}, \ldots, a_{N}\right)
$$

The equation (1) is called an ultra-discrete equation in the sense that it is a difference equation which is piecewise linear with respect to the dependent variables $x_{k}^{n}$. The s2s-OVCA includes the ECA184 $\left(n_{0}=0, v_{0}=1\right)[16]$, the FI model $\left(n_{0}=0\right)[4]$ and the s2s model $\left(n_{0}=1\right.$, $\left.v_{0}=1\right)[12]$ as its special cases.

Since the second term in the right hand side of equation (1) gives the speed of the car $k$ at the time $n$, the s2s-OVCA describes many cars running on a single lane highway in one direction, which is driven by cautious drivers requiring enough headway to go on at least for $n_{0}$ time steps before they accelerate their cars. The equation (1) also means that the car slows down immediately when its headway becomes less than its velocity. Thus the monitoring period $n_{0}$ describes asymmetry between acceleration and deceleration of the cars. It is said that the acceleration times are about five to ten times larger than the braking times [5]. 
Without loss of generality, we can assume that the cars are arrayed in numerical order, $x_{1}^{0}<x_{2}^{0}<\cdots<x_{K}^{0}$, which is also assumed throughout below. Then the number of empty cells between the cars $k$ and $k+1$ for any $k$ is always non-negative, i.e.,

$$
x_{k+1}^{n}-x_{k}^{n}-1:=\Delta x_{k}^{n}-1 \geq 0 .
$$

It is obvious that the inequality holds for $n=0$. We assume that the inequality holds up to some $n$, as the induction hypothesis. The induction hypothesis as well as the definition of min assure the inequality

$$
0 \leq \min \left(\min _{n^{\prime}=0}^{n_{0}}\left(\Delta x_{k}^{n-n^{\prime}}-1\right), v_{0}\right) \leq \Delta x_{k}^{n}-1
$$

for any $k$. Using equation (1), we get an expression of $\Delta x_{k}^{n}$ as

$$
\begin{aligned}
\Delta x_{k}^{n+1}-1 & =\Delta x_{k}^{n}-1+\min \left(\min _{n^{\prime}=0}^{n_{0}}\left(\Delta x_{k+1}^{n-n^{\prime}}-1\right), v_{0}\right)-\min \left(\min _{n^{\prime}=0}^{n_{0}}\left(\Delta x_{k}^{n-n^{\prime}}-1\right), v_{0}\right) \\
& =\min \left(\min _{n^{\prime}=0}^{n_{0}}\left(\Delta x_{k+1}^{n-n^{\prime}}-1\right), v_{0}\right)+\left[\Delta x_{k}^{n}-1-\min \left(\min _{n^{\prime}=0}^{n_{0}}\left(\Delta x_{k}^{n-n^{\prime}}-1\right), v_{0}\right)\right] .
\end{aligned}
$$

The inequality (3) and the equation (4) show that the inequality (2) holds for $n+1$. The inequality (2) means that both overtake and clash are prohibited by the s2s-OVCA.

We should note that the s2s-OVCA is obtained from a difference equation by a limiting procedure named ultradiscretization [14], which generates a piecewise-linear equation from a difference equation via the limit formula

$$
\lim _{\delta x \rightarrow+0} \delta x \log \left(\sum_{k=0}^{N} b_{k} \mathrm{e}^{a_{k} / \delta x}\right)=\max \left(a_{0}, a_{1}, a_{2}, \ldots, a_{N}\right)=: \max _{k=0}^{N}\left(a_{k}\right),
$$

where arbitrary numbers $b_{k}$ must be positive. The equation (5) is rewritten as

$$
\lim _{\delta x \rightarrow+0} \delta x \log \left(\sum_{k=0}^{N} b_{k} \mathrm{e}^{-a_{k} / \delta x}\right)^{-1}=\min _{k=0}^{N}\left(a_{k}\right),
$$

for $\min \left(a_{0}, a_{1}, a_{2}, \ldots, a_{N}\right)=-\max \left(-a_{0},-a_{1},-a_{2}, \ldots,-a_{N}\right)$.

For the sake of convenience in the calculation below, we introduce two parameters, $x_{0}$ and $\delta t$, in the s2s-OVCA

$$
x_{k}^{n+1}=x_{k}^{n}+\min \left(\min _{n^{\prime}=0}^{n_{0}}\left(\Delta x_{k}^{n-n^{\prime}}\right)-x_{0}, v_{0} \delta t\right)=: x_{k}^{n}+v_{\mathrm{opt}}^{\mathrm{u}}\left(\Delta_{\mathrm{eff}} x_{k}^{n}\right) \delta t,
$$

where $\Delta_{\text {eff }} x_{k}^{n}:=\min _{n^{\prime}=0}^{n_{0}}\left(\Delta x_{k}^{n-n^{\prime}}\right)$. The parameters $x_{0}$ and $\delta t$ are the length of a cell, which corresponds to the space occupied by a single car or the length of a car itself in the shortest case imaginable, and the discrete time-step, respectively. For simulation of highway traffics, the length of a cell $x_{0}$ and the discrete time-step $\delta t$ are usually chosen as $x_{0}=7.5 \mathrm{~m}$ and $v_{0}=5 \times \frac{x_{0}}{\delta t}$ [5]. But we do not consider specific values of the parameters in the s2s-OVCA and regard them as generic. The two parameters $x_{0}$ and $\delta t$ were set to be unity in equation (1). Introduction of $x_{0}$ into the inequality (3) gives

$$
\Delta x_{k}^{n}-x_{0} \geq 0
$$

for any $n$ and $k$, which is shown by induction with the aid of an inequality

$$
0 \leq \min \left(\min _{n^{\prime}=0}^{n_{0}}\left(\Delta x_{k}^{n-n^{\prime}}-x_{0}\right), v_{0} \delta t\right) \leq \Delta x_{k}^{n}-x_{0}
$$


and an expression of $\Delta x_{k}^{n}$ derived from equation (6)

$$
\Delta x_{k}^{n+1}-x_{0}=\min \left(\min _{n^{\prime}=0}^{n_{0}}\left(\Delta x_{k+1}^{n-n^{\prime}}-x_{0}\right), v_{0}\right)+\left[\Delta x_{k}^{n}-x_{0}-\min \left(\min _{n^{\prime}=0}^{n_{0}}\left(\Delta x_{k}^{n-n^{\prime}}-x_{0}\right), v_{0}\right)\right]
$$

that correspond equations (3) and (4), respectively.

Since we have the inequality (7) for the headway $\Delta x_{k}^{n}-x_{0}$, the effective headway $\Delta_{\text {eff }} x_{k}^{n}-x_{0}$ is also always non-negative, $\Delta_{\text {eff }} x_{k}^{n}-x_{0} \geq 0$, for any $k$. With the aid of the identity

$$
\min (A, B)=A-\max (0, A-B)=\max (0, A)-\max (0, A-B)
$$

for any $A \geq 0$, the optimal velocity function $v_{\mathrm{opt}}^{\mathrm{u}}(x) \delta t:=\min \left(x-x_{0}, v_{0} \delta t\right)$ in the s2s-OVCA is expressed as

$$
v_{\mathrm{opt}}^{\mathrm{u}}(x) \delta t=\max \left(0, x-x_{0}\right)-\max \left(0, x-x_{0}-v_{0} \delta t\right),
$$

for any $x>0$. It is given by the ultradiscrete limit $\delta x \rightarrow+0$ of a function

$$
v_{\mathrm{opt}}^{\mathrm{d}}(x) \delta t=\delta x \log \left[\frac{\frac{1+\mathrm{e}^{\left(x-x_{0}\right) / \delta x}}{1+\mathrm{e}^{-x_{0} / \delta x}}}{\frac{1+\mathrm{e}^{\left(x-x_{0}-v_{0} \delta t\right) / \delta x}}{1+\mathrm{e}^{-\left(x_{0}+v_{0} \delta t\right) / \delta x}}}\right],
$$

which is an inverse-ultradiscretization of the optimal velocity function $v_{\mathrm{opt}}^{\mathrm{u}}$. Note that we have introduced arbitrary coefficients so as to make $v_{\mathrm{opt}}^{\mathrm{d}}(0)=0$. In a similar way to the above calculation, an inverse-ultradiscretization of the effective interval $\Delta_{\text {eff }}^{u} x_{k}^{n}$ is also obtained as

$$
\Delta_{\text {eff }}^{\mathrm{d}} x_{k}^{n}:=\delta x \log \left(\sum_{n^{\prime}=0}^{n_{0}} \frac{\mathrm{e}^{-\Delta x_{k}^{n-n^{\prime}} / \delta x}}{n_{0}+1}\right)^{-1} .
$$

Therefore an inverse-ultradiscretization of the us2s-OVCA is given by $x_{k}^{n+1}=x_{k}^{n}+v_{\mathrm{opt}}^{\mathrm{d}}\left(\Delta_{\text {eff }}^{\mathrm{d}} x_{k}^{n}\right) \delta t$, which is explicitly written as

$$
\begin{aligned}
x_{k}^{n+1}= & x_{k}^{n}+\delta x\left\{\log \left[1+\left(\sum_{n^{\prime}=0}^{n_{0}} \frac{\mathrm{e}^{-\left(\Delta x_{k}^{n-n^{\prime}}-x_{0}\right) / \delta x}}{n_{0}+1}\right)^{-1}\right]-\log \left(1+\mathrm{e}^{-x_{0} / \delta x}\right)\right. \\
& \left.-\log \left[1+\left(\sum_{n^{\prime}=0}^{n_{0}} \frac{\mathrm{e}^{-\left(\Delta x_{k}^{n-n^{\prime}}-x_{0}-v_{0} \delta t\right) / \delta x}}{n_{0}+1}\right)^{-1}\right]+\log \left(1+\mathrm{e}^{-\left(x_{0}+v_{0} \delta t\right) / \delta x}\right)\right\} .
\end{aligned}
$$

In other words, the s2s-OVCA is given by the ultradiscrete limit $\delta x \rightarrow+0$ of the above difference equation (8).

Since equation (8) is rewritten as

$$
\begin{aligned}
\frac{x_{k}^{n+1}-x_{k}^{n}}{\delta t}= & \delta x\left\{-\frac{1}{\delta t}\left(\log \left[1+\left(\sum_{n^{\prime}=0}^{n_{0}} \frac{\mathrm{e}^{-\left(\Delta x_{k}^{n-n^{\prime}}-x_{0}-v_{0} \delta t\right) / \delta x}}{n_{0}+1}\right)^{-1}\right]\right.\right. \\
& \left.-\log \left[1+\left(\sum_{n^{\prime}=0}^{n_{0}} \frac{\mathrm{e}^{-\left(\Delta x_{k}^{n-n^{\prime}}-x_{0}\right) / \delta x}}{n_{0}+1}\right)^{-1}\right]\right) \\
& \left.+\frac{\log \left(1+\mathrm{e}^{-\left(x_{0}+v_{0} \delta t\right) / \delta x}\right)+\log \left(1+\mathrm{e}^{-x_{0} / \delta x}\right)}{\delta t}\right\} \\
= & v_{0}\left(1+\sum_{n^{\prime}=0}^{n_{0}} \frac{\mathrm{e}^{-\left(\Delta x_{k}^{n-n^{\prime}}-x_{0}\right) / \delta x}}{n_{0}+1}\right)^{-1}-v_{0}\left(1+\mathrm{e}^{x_{0} / \delta x}\right)^{-1}+O(\delta t),
\end{aligned}
$$


the above difference equation (8) goes to an integral-differential equation in the continuum limit $\delta t \rightarrow 0$ as follows

$$
\frac{\mathrm{d} x_{k}}{\mathrm{~d} t}=v_{0}\left(1+\frac{1}{t_{0}} \int_{0}^{t_{0}} \mathrm{e}^{-\left(\Delta x_{k}\left(t-t^{\prime}\right)-x_{0}\right) / \delta x} \mathrm{~d} t^{\prime}\right)^{-1}-v_{0}\left(1+\mathrm{e}^{x_{0} / \delta x}\right)^{-1},
$$

where $t_{0}:=n_{0} \delta t, \frac{\mathrm{d} x_{k}}{\mathrm{~d} t}=\lim _{\delta t \rightarrow 0} \frac{x_{k}^{n+1}-x_{k}^{n}}{\delta t}$ and

$$
\lim _{\delta t \rightarrow 0} \sum_{n^{\prime}=0}^{n_{0}} \frac{\mathrm{e}^{-\left(\Delta x_{k}^{n-n^{\prime}}-x_{0}\right) / \delta x}}{n_{0}+1}=\frac{1}{t_{0}} \int_{0}^{t_{0}} \mathrm{e}^{-\left(\Delta x_{k}\left(t-t^{\prime}\right)-x_{0}\right) / \delta x} \mathrm{~d} t^{\prime} .
$$

In terms of an optimal velocity function and an effective distance

$$
\begin{aligned}
& v_{\text {opt }}(x):=v_{0}\left(\frac{1}{1+\mathrm{e}^{-\left(x-x_{0}\right) / \delta x}}-\frac{1}{1+\mathrm{e}^{x_{0} / \delta x}}\right), \\
& \Delta_{\text {eff }} x_{k}(t):=\delta x \log \left(\frac{1}{t_{0}} \int_{0}^{t_{0}} \mathrm{e}^{-\Delta x_{k}\left(t-t^{\prime}\right) / \delta x} \mathrm{~d} t^{\prime}\right)^{-1},
\end{aligned}
$$

the above integral-differential equation is expressed as

$$
\frac{\mathrm{d} x_{k}}{\mathrm{~d} t}=v_{\text {opt }}\left(\Delta_{\text {eff }} x_{k}(t)\right) .
$$

Since the effective distance $\Delta_{\text {eff }} x_{k}(t)$ goes to $\Delta x_{k}(t)$ in the limit below

$$
\Delta x_{k}\left(t-t_{0}\right)=\lim _{h \rightarrow t_{0}} \delta x \log \left(\frac{1}{t_{0}-h} \int_{h}^{t_{0}} \mathrm{e}^{-\Delta x_{k}\left(t-t^{\prime}\right) / \delta x} \mathrm{~d} t^{\prime}\right)^{-1},
$$

this integral-differential equation is an extension of the Newell model [8]

$$
\frac{\mathrm{d} x_{k}}{\mathrm{~d} t}=v_{\mathrm{opt}}\left(\Delta x_{k}\left(t-t_{0}\right)\right),
$$

which is a car-following model dealing with retarded adaptation to the optimal velocity determined by the headway in the past.

Replacement of $t$ with $t+t_{0}$ in equation (10) and the Taylor expansion of $\dot{x}_{k}\left(t+t_{0}\right)=v_{k}\left(t+t_{0}\right)$ yield

$$
v_{\text {opt }}\left(\Delta x_{k}(t)\right)=v_{k}\left(t+t_{0}\right)=v_{k}(t)+\frac{\mathrm{d} v_{k}}{\mathrm{~d} t} \cdot t_{0}+\frac{1}{2} \frac{\mathrm{d}^{2} v_{k}}{\mathrm{~d} t^{2}} \cdot t_{0}^{2}+\cdots,
$$

which is equivalent to

$$
\frac{\mathrm{d} v_{k}}{\mathrm{~d} t}+\frac{1}{2} \frac{\mathrm{d}^{2} v_{k}}{\mathrm{~d} t^{2}} \cdot t_{0}+\cdots=\frac{1}{t_{0}}\left(v_{\text {opt }}\left(\Delta x_{k}(t)\right)-v_{k}(t)\right) .
$$

The equation of motion of the OV model

$$
\frac{\mathrm{d} v_{k}}{\mathrm{~d} t}=\frac{1}{t_{0}}\left(v_{\mathrm{opt}}\left(\Delta x_{k}(t)\right)-v_{k}(t)\right)
$$

is given by neglecting the higher order terms in the left hand side of the equation (11).

The discussion shown above in this section shows how the inverse ultradiscretization and the time continuous limit connect the s2s model and the Newell model, which approximates the OV model, through the s2s-OVCA. 

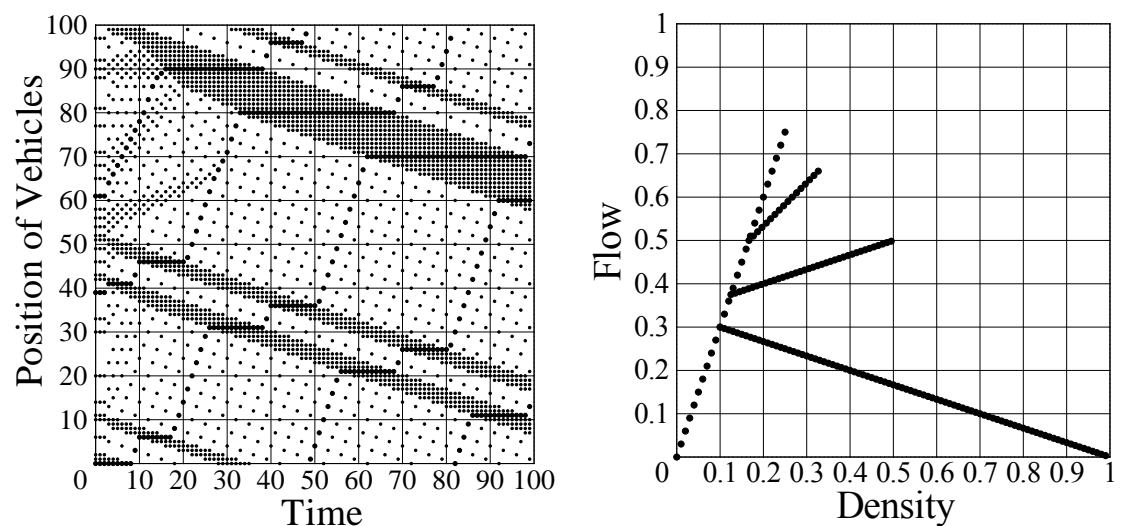

Figure 1. The spatio-temporal pattern (left) and the flow-density relation (right) of the s2s-OVCA [9].

\section{Flow-density relation}

Fig. 1 gives typical examples of the spatio-temporal pattern showing jams and the flow-density relation of the s2s-OVCA [9]. In the numerical calculation, the periodic boundary condition is imposed and the length of the circuit $L$, which is the same as the number of all the cells, is fixed at $L=100$. The maximum velocity $v_{0}$ and the monitoring period $n_{0}$ are $v_{0}=3$ and $n_{0}=2$. The number of the cars $K$ in the spatio-temporal pattern is set at $K=30$.

The spatio temporal pattern shows the trajectories of the cars. As we can see, irregular motion of cars is observed in the early stage of the time evolution, $0 \leq n \leq 30$, where $n$ is the time. But after that, the flow of the cars become stationary in the sense that length of the jam is almost constant and that cars with intermediate speeds appear only temporarily.

The flows $Q$ in the flow-density relation are computed by averaging over the time period $800=n_{\mathrm{i}} \leq n \leq n_{\mathrm{f}}=1000$,

$$
Q:=\frac{1}{\left(n_{f}-n_{i}+1\right) L} \sum_{k=1}^{K} \sum_{n=n_{i}}^{n_{f}} v_{k}^{n}, \quad v_{k}^{n}:=x_{k}^{n+1}-x_{k}^{n},
$$

in which the traffic is expected to be stationary in the above mentioned sense. The car density $\rho$ is given by $\rho:=\frac{K}{L}$. As we have mentioned before, the flow-density relation of the s2s-OVCA is piecewise linear and flipped- $\lambda$ shaped with several metastable slow branches.

The flow-density relation shown above is derived by admitting the features of the flow of the s2s-OVCA. Namely, the flow of the s2s-OVCA goes to one of the stationary flows in the long run. The stationary flows consist of the free flow in which all the cars run at the top speed $v_{0}$ and the slow flows that always contain slow cars running at the minimum speed $v_{\min }^{\infty}$, $0 \leq v_{\min }^{\infty}<v_{0}$, which remains constant. Formation of the line of slow cars corresponds to that of traffic jam. In the slow flows, lengths of the jams are almost constant and fluctuate periodically. Our previous paper [15] gives a set of such stationary flows.

First we shall deal with the free flow and its flow-density relation. Since all the cars run at the top speed, $v_{k}^{n}=v_{0}, \forall k$, all the headways respectively remain constant, $\Delta x_{k}^{n+1}=\Delta x_{k}^{n} \geq v_{0}+1$, $\forall k$. Hence the flow $Q$ is also constant in the future. Using the definition of the flow (12) with $n_{\mathrm{i}}=n_{\mathrm{f}}=n$, we have

$$
Q=\frac{1}{L} \sum_{k=1}^{K} v_{0}=\rho v_{0}
$$

which gives the straight line in the flow-density relation with a positive inclination that is equal 


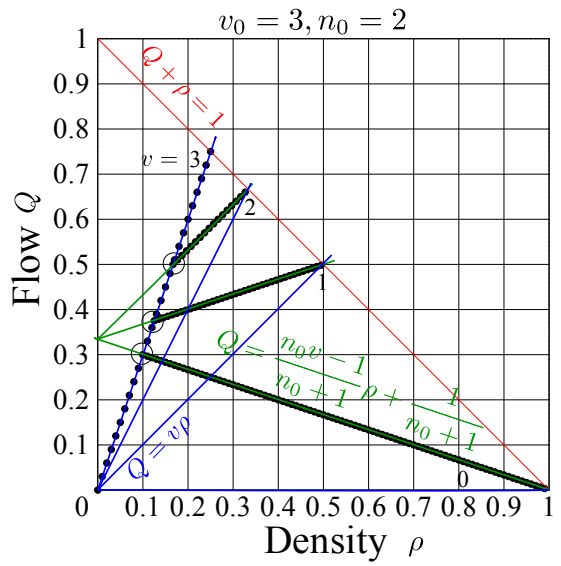

Figure 2. The formula for the flow-density relation of the s2s-OVCA [15].

to the top speed $v_{0}$. For example in Fig. 2, the flow-density relation of the free flow which is labeled with $v=3$ is $Q=3 \rho$, since $v_{0}=3$ in this case.

Next we shall consider the slow flows and their flow-density relations. Let us see a specific solution of the s2s-OVCA starting from the following initial configuration

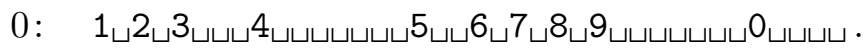

Note that the number 0 at the leftmost shows the time. The digits and the blank symbols $\sqcup$ in the above configuration mean the indices of the cars and the empty cells, respectively. Thus the number of the cars $K$ is 10 and the length of the circuit $L$ is 38 in this case. We set the monitoring period $n_{0}$ at 2 . The speed of the cars 4,9 and 0 is 3 , which is the top speed $v_{0}$ of this case. The speed of the car 5 is 2, whose headway is also 2. All the other cars' speeds are 1 , whose headways are also 1 except for the car 3 . Thus the headways of the cars in tha past have nothing to do with the motion of the cars in the future except for the car 3 . The headway of the car 3 at the time -1 is set to be 1 .

Out of the above initial configuration (13), the equation (1) generates flow of vehicles as follows

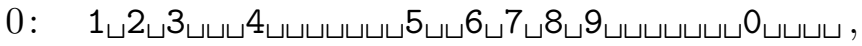

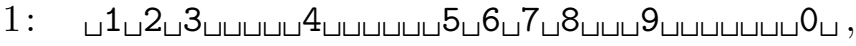

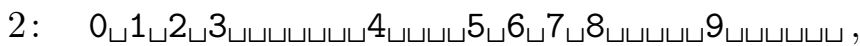

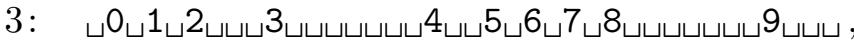

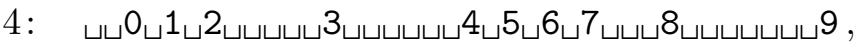

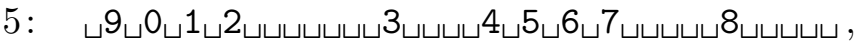

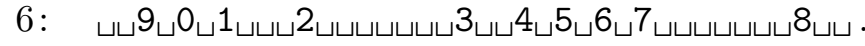

Note that the minimum speed of the cars $v_{\text {min }}^{\infty}$ is 1 in the flow above. We notice that the configuration at the time 3 is obtained by moving all the cells of the initial configuration one cell rightward as well as changing the car indices $k$ to $k-1$ modulo 10 . The configuration at the time 6 is also obtained by doing the same shifts and changes of car indices to the configuration at the time 3. In this sense, the above flow is a periodic motion of cars whose period is 3 in this case. The length of the jam, or the number of the cars running at the minimum speed, is thus almost constant. Intermediate speeds also appear but only temporarily. That is why we call them stationary flows of the s2s-OVCA. Roughly speaking, the slow flow we shall deal with is the stationary flow of the type shown above. The density of the cars $\rho$ and the average flow $Q$ 


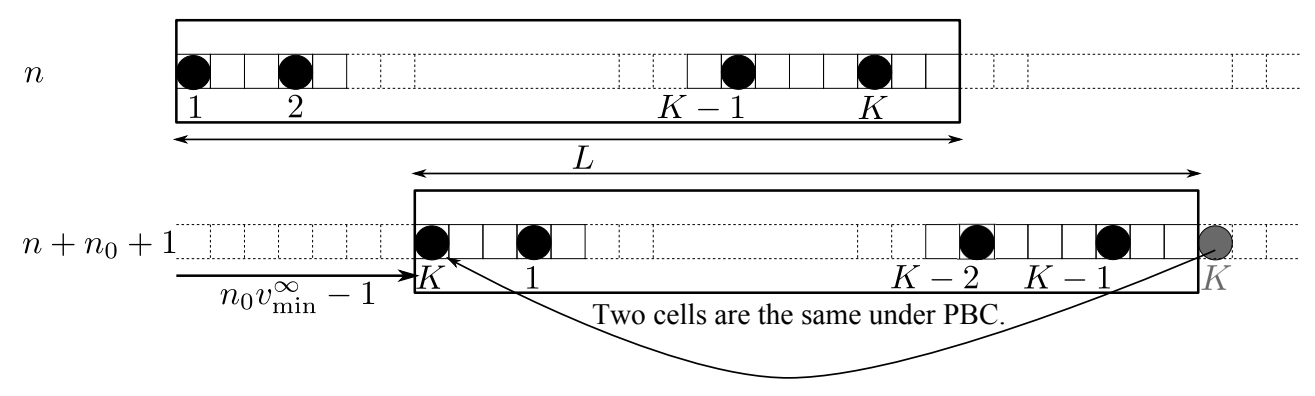

Figure 3. The slow flow of the s2s-OVCA. Cars in the cells drawn by dashed lines are omitted except for the car 1 at the time $n+n_{0}+1$.

over the period, or the $n_{0}+1=3$ steps, are calculated as

$$
\begin{aligned}
& \rho=\frac{K}{L}=\frac{5}{19}, \\
& Q=\frac{1}{\left(n_{0}+1\right) L} \sum_{k=0}^{K} \sum_{n^{\prime}=0}^{n_{0}} v_{k}^{n^{\prime}}=\frac{6+3+3+5+9+4+3+3+3+9}{3 \times 38}=\frac{8}{19},
\end{aligned}
$$

which will be verified with the formula we shall derive shortly.

Let us consider such slow flows as we have seen above as the specific solutions in a more general manner. Fig. 3 shows configurations of a slow flow at times $n$ and $n+n_{0}+1$. Since we employ the periodic boundary condition, two cells containing the car $K$ are identified. As a property of the slow flow, we assume that the slow flow is periodic in the sense that the configuration at the time $n+n_{0}+1$, which is shown in the box in Fig. 3, is given by the rightward displacement of the entire configuration at the time $n$ in the box by $n_{0} v_{\min }^{\infty}-1$ cells. The flow provided by this displacement of the entire configuration in $n_{0}+1$ time steps is $\frac{n_{0} v_{\min }^{\infty}-1}{n_{0}+1} \rho$. For example, the rightward displacement mentioned above for the slow flow in Fig. 3 is $2 \times 1-1=1$, which agrees with the observation before. The set of stationary flows given in [15] has the property of the slow flow we here assume.

Here we should note that the leftward displacement of the car $K$ by $L$ cells, namely whole the circuit length, which is fictitiously introduced to make the shifted initial configuration from the real configuration at the time $n_{0}+1$ in the sense that the numerical order of the car arrays is maintained. In order to compensate the underestimation of the flow brought about by this leftward displacement, we have to add the flow corresponding to the rightward displacement of the car $K$ by $L$ cells in $n_{0}+1$ time steps, $\frac{1}{\left(n_{0}+1\right) L} \cdot L=\frac{1}{n_{0}+1}$. Thus the flow of the slow flow with the minimum speed $v_{\min }^{\infty}$ is given by

$$
Q=\frac{n_{0} v_{\min }^{\infty}-1}{n_{0}+1} \rho+\frac{1}{n_{0}+1}, \quad 0 \leq v_{\min }^{\infty}<v_{0}
$$

For example, substitution of $\rho=\frac{5}{19}, n_{0}=2$ and $v_{\min }^{\infty}=1$ into equation (14) yields

$$
Q=\frac{2 \times 1-1}{2+1} \times \frac{5}{19}+\frac{1}{2+1}=\frac{8}{19}
$$

which agrees with the flow $Q=\frac{8}{19}$ for the slow flow given above as an specific solution. The formula (14) agrees with the flow-density relation given by numerical experiments, as we can see in Fig. 2. Three branches labeled with $v=2,1$ and 0 are the flow-density relations with the minimum speeds $v_{\text {min }}^{\infty}=v$ in Fig. 2. Roughly speaking, the traffic that forms the branch corresponding to $v_{\text {min }}^{\infty}$ consists of groups of cars running at the top speed $v_{0}$ and other groups of cars running at the minimum speed $v_{\mathrm{min}}^{\infty}$, as the specific solution evolving from the initial 
configuration (13) has shown. A set of specific solutions that correspond to the branches in the fundamental diagram is given in [15].

The maximum density $\rho_{\max }\left(v_{\text {min }}^{\infty}\right)$ that allows the minimum speed to be $v_{\text {min }}^{\infty}$ is

$$
\rho_{\max }\left(v_{\min }^{\infty}\right)=\frac{1}{v_{\min }^{\infty}+1} .
$$

The flow $Q\left(\rho_{\max }\left(v_{\min }^{\infty}\right)\right)$ corresponding to the maximum density $\rho_{\max }\left(v_{\min }^{\infty}\right)$ is then given by

$$
Q\left(\rho_{\max }\left(v_{\min }^{\infty}\right)\right)=\rho_{\max }\left(v_{\min }^{\infty}\right) v_{\min }^{\infty} .
$$

Since the two equations (15) and (16) holds at the same time, they leads to $Q\left(\rho_{\max }\left(v_{\min }^{\infty}\right)\right)+$ $\rho_{\max }\left(v_{\text {min }}^{\infty}\right)=1$. Thus all the end points of the branches must be on the line

$$
Q+\rho=1 .
$$

The branching point, or the minimum density, of the flow-density relation of the slow flow corresponding to the minimum speed $v_{\text {min }}^{\infty}$ is determined by the intersection of the flow density relations of the free flow and the slow flow

$$
\rho_{\min }\left(v_{\min }^{\infty}\right)=\frac{1}{n_{0}\left(v_{0}-v_{\min }^{\infty}\right)+v_{0}+1} .
$$

In Fig. 2, the branching points corresponding to $v_{\text {min }}^{\infty}=2,1$ and 0 are encircled with small circles, which agree with the above formula (18). The density of the cars $\rho$ needs to be sufficiently large so as to form the slow flow with the minimum speed $v_{\text {min }}^{\infty}$. The branching point gives the lower bound of such density.

Since the s2s-OVCA (1) is a deterministic CA, the initial configuration determines the final state. Its numerical simulation is very robust against, or more precisely speaking, free from numerical errors. Thus all the stationary flows including the free flow and the slow flows beyond the minimum density $\rho_{\min }\left(v_{\min }^{\infty}\right)$ are stable in the numerical simulation. That is why we were able to obtain the flow-density relation with several "metastable" states consisted by the free flow and the slow flows beyond the minimum density, as was shown in Figs. 1 and 2. Though these metastable states are robust against numerical errors in simulation, but they are generally unstable against perturbation, which gives a reason of their name. For example, when one gives a perturbation to the headways that is equal to the minimum speed $v_{\min }^{\infty}$ in the groups of cars running at the minimum speed $v_{\text {min }}^{\infty}$, a car with a velocity that is less than $v_{\text {min }}^{\infty}$ appears. And such a perturbed car generally becomes a seed of a group of cars running at a velocity slower than $v_{\min }^{\infty}$, which eventually slows down whole the traffic. That is why we call these states metastable, except for the slow line with $v_{\min }^{\infty}=0$, which we cannot make slower.

When the monitoring period $n_{0}$ is zero, all of the slow flows (14) goes to the line of the end points of the metastable branches (17). Thus the monitoring period plays an essential role in the formation of the metastable states in the flow-density relation. Thus it could be determined by comparing the flow-density relation of the s2s-OVCA and observed ones.

\section{Summary}

We have shown an inverse ultradiscretization from the s2s-OVCA (1) to an integral-differential equation (9), which is an extension of the Newell model (10). Since the Newell model [8] and the s2s-OVCA [9] are extended models of the OV [1] and the s2s models [12] respectively, the s2s-OVCA is interpreted as a CA-type hybrid of the OV and the s2s models.

Using the features of the stationary flows observed in the numerical experiments, we have derived the flow-density relations of the stationary flow of the s2s-OVCA. The flow-density 
relations of the s2s-OVCA were numerically obtained [9] and then derived by use of a set of stationary flows [15].

Since the s2s-OVCA is a deterministic CA, the model is suitable for a simulation with a much bigger system size than the length of the circuit, $L=100$, in our numerical simulation. We expected that it would be sufficient to capture the characteristics of stationary flows of the s2sOVCA on the circuit, which is determined by the density of cars and initial configuration. In order to observe finite-size effects of open boundaries, for example, we expect that the s2s-OVCA will be a good tool.

The s2s-OVCA has several types of monotonicity in its time evolution, which extend the results shown for the $n_{0}=1$ case [13]. We expect that the monotonicity determines the relaxation to the stationary flow from the initial configuration as well as the property of the stationary flow we assume here. We hope that results on the relaxation to stationary flows and the monotonicity in the time evolution of the s2s-OVCA will be reported soon.

\section{Acknowledgments}

One of the authors (HU) is grateful to K. Oguma for the previous collaboration.

\section{References}

[1] Bando M., Hasebe K., Nakayama A., Shibata A., Sugiyama Y., Dynamical model of traffic congestion and numerical simulation, Phys. Rev. E 51 (1995), 1035-1042.

[2] Barlovic R., Santen L., Schadschneider A., Schreckenberg M., Metastable states in cellular automata for traffic flow, Eur. Phys. J. B 5 (1998), 793-800, cond-mat/9804170.

[3] Chowdhury D., Santen L., Schadschneider A., Statistical physics of vehicular traffic and some related systems, Phys. Rep. 329 (2000), 199-329, cond-mat/0007053.

[4] Fukui M., Ishibashi Y., Traffic flow in 1D cellular automaton model including cars moving with high speed, J. Phys. Soc. Japan 65 (1996), 1868-1870.

[5] Helbing D., Traffic and related self-driven many-particle systems, Rev. Modern Phys. 73 (2001), 1067-1141, cond-mat/0012229.

[6] Helbing D., Schreckenberg M., Cellular automata simulating experimental properties of traffic flow, Phys. Rev. E 59 (1999), R2505-R2508, cond-mat/9812300.

[7] Kanai M., Isojima S., Nishinari K., Tokihiro T., Ultradiscrete optimal velocity model: a cellular-automaton model for traffic flos and linear instability of high-flux traffic, Phys. Rev. E $\mathbf{7 9}$ (2009), 056108, 8 pages, arXiv:0902.2633.

[8] Newell G.F., Nonlinear effects in the dynamics of car flowing, Operations Res. 9 (1961), 209-229.

[9] Oguma K., Ujino H., A hybrid of the optimal velocity and the slow-to-start models and its ultradiscretization, JSIAM Lett. 1 (2009), 68-71, arXiv:0908.3377.

[10] Takahashi D., Matsukidaira J., On a discrete optimal velocity model and its continuous and ultradiscrete relatives, JSIAM Lett. 1 (2009), 1-4, arXiv:0809.1265.

[11] Takahashi D., Satsuma J., A soliton cellular automaton, J. Phys. Soc. Japan 59 (1990), 3514-3519.

[12] Takayasu M., Takayasu H., 1/f noise in a traffic model, Fractals 1 (1993), 860-866.

[13] Tian R., The mathematical solution of a cellular automaton model which simulates traffic flow with a slowto-start effect, Discrete Appl. Math. 157 (2009), 2904-2917.

[14] Tokihiro T., Takahashi D., Matsukidaira J., Satsuma J., From soliton equations to integrable cellular automata through a limiting procedure, Phys. Rev. Lett. 76 (1996), 3247-3250.

[15] Ujino H., Yajima T., Exact solutions and flow-density relations for a cellular automaton variant of the optimal velocity model with the slow-to-start effect, J. Phys. Soc. Japan 81 (2012), 124005, 8 pages, arXiv:1210.7562.

[16] Wolfram S. (Editor), Theory and applications of cellular automata, Advanced Series on Complex Systems, Vol. 1, World Scientific Publishing Co., Singapore, 1986. 